\title{
Study on Dispersion Stability and Friction Characteristics of C60 Nanomicrosphere Lubricating Additives for Improving Cutting Conditions in Manufacturing Process
}

\author{
Jing-Shan Huang $\mathbb{D},{ }^{1,2}$ Hao Sun, ${ }^{3}$ Xi Wang, ${ }^{1,2}$ Bin-Qiang Chen, ${ }^{1,2}$ and Bin Yao $\mathbb{D}^{1,2}$ \\ ${ }^{1}$ School of Aerospace Engineering, Xiamen University, Xiamen 361005, China \\ ${ }^{2}$ Shenzhen Research Institute of Xiamen University, Shenzhen 518000, China \\ ${ }^{3}$ AECC Harbin Dongan Engine Co., Ltd., Harbin 150060, China \\ Correspondence should be addressed to Bin Yao; aeroiiet@126.com
}

Received 13 April 2021; Accepted 5 October 2021; Published 19 October 2021

Academic Editor: Yuqing Zhou

Copyright (c) 2021 Jing-Shan Huang et al. This is an open access article distributed under the Creative Commons Attribution License, which permits unrestricted use, distribution, and reproduction in any medium, provided the original work is properly cited.

\begin{abstract}
Antifriction lubrication is an important research hotspot in the manufacturing field. A high-performance lubricating additive is of great significance for condition monitoring in the metal cutting process system. To improve cutting conditions in manufacturing process, we study the dispersion stability and tribological properties of fullerene nanoparticles in HM32 antiwear lubricating fluid. Fullerene nanoparticles are fully integrated into HM32 antiwear lubricating fluid by electromagnetic stirring and ultrasonic oscillation. The dispersion stability of fullerene nanoparticles in HM32 antiwear lubricating fluid was comprehensively studied by microscope scanning experiment, static sedimentation experiment, and absorption experiment. The four-ball friction experiment was operated to investigate the extreme pressure property and tribological property of lubricating fluids with fullerene concentration ranging from $100 \mathrm{ppm}$ to $1000 \mathrm{ppm}$. The results show that fullerene nanoparticle can significantly improve the extreme pressure property and wear resistance of HM32 basic lubricating fluid. Meanwhile, we found that an excessively high concentration of fullerene nanoparticles will increase the friction and wear of the four-ball friction pair. The best concentration of fullerene nanoparticles is $200 \mathrm{ppm}$. When the fullerene concentration reaches $200 \mathrm{ppm}$, the maximum nonsintering load is significantly increased, and the friction coefficient and the steel ball wear scar diameter are significantly reduced.
\end{abstract}

\section{Introduction}

With the development of modern industry, the requirements for precision, efficiency, and reliability of mechanical equipment have become increasingly strict. The lubrication technology is of significant importance in the mechanical equipment field. The energy waste caused by friction and wear has seriously hindered the rapid development of the industry. The economic losses caused by the failure of mechanical parts due to friction and wear account for a large proportion of the GDP [1]. Meanwhile, high-performance lubricating additives can promote the stable operation of the cutting process system. It is of great significance to the condition monitoring in the metal cutting process [2]. Therefore, reducing the friction and wear in mechanical equipment is a subject of great social significance.

In recent years, domestic and foreign scholars have conducted extensive researches on the friction reduction and operational stability of the manufacturing process. Based on learning machine methods [3-6], signal processing algorithms [7-9], and simulation modelling technologies $[10,11]$, scholars have explored tool wear condition detection [12-16], bearing condition monitoring [17-21], ball screw detection [22, 23], mechanical signature analysis $[24,25]$, nanoparticle cutting fluid $[26,27]$, and so on in the cutting process system. In particular, improving lubrication technology is the most effective way to reduce the friction and wear in the manufacturing process. Countries all over the world attach great importance to the research of antiwear 
and antifriction technologies. For most mechanical equipment, adding lubricant between friction surfaces is the main method to reduce friction and wear. Lubricants can not only reduce the friction coefficient and slow down wear, but also continuously absorb heat from the friction surface to prevent damage to parts and tools. In addition, lubricants can wash away metal chips and dust generated by friction and wear. However, the effectiveness of traditional lubricants is limited. It is necessary to add various additives in the lubricant to enhance the lubricity [28]. In the metal cutting process, nanomicrosphere lubricating additives can be applied in the cutting fluid to enhance the lubrication and antiwear properties of the cutting fluid [29]. By fully mixing the nanomicrosphere lubricating additives and the cutting fluid, the cutting fluid containing the nanoparticles is added to the cutting fluid circulation system. Nanoparticles infiltrate into the contact surface between the cutting tool and the workpiece or chips with the cutting fluid, turning the sliding friction between the tool and the workpiece into rolling/ sliding mixed friction. Meanwhile, it can reduce the direct contact between the tool-chip peak points, decrease the toolchip bonding friction and chip fibrosis, and reduce the cutting force and cutting heat in the manufacturing process. Therefore, the application of nanomicrosphere lubricating additives can reduce cutting tool wear, delay tool life, and improve production efficiency in the mechanical manufacturing process. Nowadays, using nanoparticles as lubricant additives has become a research hotspot in the lubrication field.

Nanomaterial lies in the transition zone between macroscopic matter and microscopic atoms and molecules, which make it exhibit many distinctive properties [30]. Nanoparticle has the characteristics of surface effect, small size effect, quantum size effect, and macroscopic quantum tunneling effect. Nanoparticles are mainly used as friction reducers, antiwear agents, and extreme pressure agents in lubrication [31-35]. They have the potential to replace traditional lubricant additives.

Some lubrication mechanisms have been proposed in the literature. When nanoparticles are used as lubricant additives, it led to the following effects:

(1) Rolling effect: nanoparticles are easy to roll when doing relative movement between friction pairs. It turns the sliding friction into rolling friction between the surfaces of the friction pair. The generated heat is reduced by reducing the contact area. Meanwhile, the nanoparticles play a supporting role and improve the carrying capacity [36].

(2) Protective film: the nanoparticles are adsorbed to the surface of the friction pair in the friction and wear process due to the high ratio of surface area-to-volume. The exchange of material molecules occurs between the friction pair and the lubricating material under the tribochemical reaction [37]. Therefore, a reaction film with low shear strength and high hardness is formed. The reaction film prevents direct contact between the friction pairs and improves the wear resistance.
(3) Mending effect: there are some scratches and holes on the surface of the friction pair. Nanoparticles enter these holes due to their small size. Consequently, it provides a flat surface that reduces friction and cutting temperature [38]. The mending effect is beneficial to the release of stress and the improvement of tribological properties.

(4) Polishing effect: some hard nanoparticles are polishing materials. They can process ultrasmooth surfaces with root mean square roughness (RMS) ranging from 0.1 to $1.0 \mathrm{~nm}$ [39]. Nanoparticles will also produce mechanical polishing on the surface of the friction pair during the friction process. The friction coefficient and the contact pressure of the contact surfaces are reduced after nanoparticle polishing [40].

(5) Chemical reaction: nanoparticles may also chemically react with the friction pair surface to form a lubricating film. For example, a lubricant containing $\mathrm{Cu}$ nanoadditives reacts with the steel surface to form a lubricating film composed of $\mathrm{Cu}, \mathrm{FeS}$, and FeSO4 [41].

Nanolubricants show many advantages compared to traditional lubricants [42-44]. The oil film strength of nanoparticle additives is higher than that of traditional additives [45]. The suspension density and uniformity of nanoparticle additives are also much higher than those of traditional additives [46]. In addition, nanoparticle additives are more environmentally friendly than traditional additives [47].

Nowadays, using nanoparticles as lubricant additives has become a research hotspot in the lubrication field. The research and application of nanoparticle lubricating additives in lubricating systems provide new ideas for advanced lubricating materials. In recent years, many scholars and experts have conducted a lot of research work on the dispersion stability and lubrication mechanism of nanolubricants. Imene et al. [48] used a transmission nanoindenter to observe that spherical MoS2 nanoparticles exhibit a rolling effect when the positive pressure is less than $100 \mathrm{MPa}$. It significantly improves the lubricant carrying capacity and reduces the friction coefficient and wear. Peng et al. [49] prepared $\mathrm{SiO}_{2}$ nanoparticles and diamond nanoparticles with good dispersion in lubricants and discussed their tribological properties. It is found that $\mathrm{SiO}_{2}$ nanoparticles and diamond nanoparticles are filled in the more severely worn microregions to repair the friction surface. Xia et al. [50] found that inorganic nanoparticles can form a boundary lubricant film on the friction surface under high temperature and high pressure. In addition, the nanoparticles deposited between the friction surfaces can fill the surface pits and damaged parts to reduce friction and wear. $\mathrm{Xu}$ et al. [51] prepared a water-soluble nano- $\mathrm{Cu}$ additive with an average particle size of $3.5 \mathrm{~nm}$ using in situ surface modification. Using nano-Cu particles as a waterbased lubricant additive can reduce the friction coefficient and wear rate of pure water by about $50 \%$. It shows that the nanocopper particles have excellent antifriction and 
antiwear properties. Zhao et al. [52] modified graphene with Span-80 as a dispersant to make it uniformly and stably dispersed in the base oil. It is found that graphene can improve the friction and wear performance of CGr15/ bronze friction pair. Graphene has the most obvious antiwear effect at $60^{\circ} \mathrm{C}$ and $100^{\circ} \mathrm{C}$, and the friction coefficient and wear rate are reduced by $78 \%$ and $95 \%$, respectively. GUPTA [53] used three nanofluids of aluminum oxide ( $\mathrm{Al} 2 \mathrm{O} 3)$, molybdenum disulfide (MoS2), and nanographite to optimize the cutting force, tool wear, cutting temperature, and surface finish during MQL turning of titanium alloys. PADMINI [54] studied the effect of vegetable oil-based nMoS2 nanofluid in the steel turning process. It was found that nMoS2 nanofluid can effectively reduce cutting force, temperature, tool wear, and surface roughness. Sharma et al. [55] studied the effect of Al2O3/graphene hybrid nanoparticle additives in the AISI 304 steel turning process to improve the tribological properties of lubricants. The developed hybrid nanolubricant significantly reduces tool side wear and cutting temperature. Cetin and Kilincarslan [56] used the suspension of borax-ethylene glycol and colloidal suspension of borax-ethylene glycol-nanosilver particles as cutting fluid for milling of AA7075-T6 material. It is found that borax and nanosilver additives can improve the lubrication function and heat transfer properties.

The research on the tribological properties of fullerene C60, which has a unique spherical shape and high hardness, is attracting increasing attention. Fullerene $\mathrm{C} 60$ is a molecule with a hollow cage structure composed of $60 \mathrm{C}$ atoms connected by $\mathrm{C}-\mathrm{C}$ bonds. It consists of twelve five-member rings and twenty six-member rings. Its appearance resembles a football. Its molecular diameter is about $0.71 \mathrm{~nm}$ and the inner cavity diameter is about $0.3 \mathrm{~nm}$ [57]. The unique spherical structure of fullerene (C60) gives it strong pressure resistance. Its pressure resistance is higher than that of diamond, and its microstrength is as high as $18 \mathrm{GPa}$. The hardness of C60 crystal increases with the increase of pressure, and its structure transforms to diamond structure. Under low pressure, fullerene molecules can roll on the graphite surface. Its strong intramolecular force, weak intermolecular force, and low surface energy make it promising as a high-grade lubricant for molecular rolling balls. Therefore, whether under low pressure or high pressure, fullerene C60 may become an excellent lubricant [58-60]. Hirata et al. [61] used diamond to prepare fullerene under high temperature conditions and tested the friction properties on the surface of the friction pair composed of steel balls and silicon wafers. The results show that the addition of fullerene on the surface of the friction pair exhibits good antifriction performance and antiwear performance, which is significantly better than oil-free lubrication and graphite. Yao et al. [62] used a four-ball machine to investigate the tribological properties of onion-like fullerenes. The results show that fullerenes have good antifriction properties. Its antifriction performance is better than that of carbon nanotubes, and the wear scar on the surface is lighter and more regular. Yan et al. [63] prepared the C60/C70 mixture by arc method and dispersed $\mathrm{C} 60 / \mathrm{C} 70$ in paraffin oil by solvent evaporation method. The research results on the SRV testing machine show that $\mathrm{C} 60 / \mathrm{C} 70$ nanoparticles can increase the extreme pressure load of paraffin oil by 3 times and reduce its friction coefficient by $1 / 3$, which can significantly reduce the wear of the friction pair. Hong et al. [64] synthesized a fullerene-styrene-maleic anhydride terpolymer and dissolved it in ethanolamine aqueous solution. The results of the four-ball friction tester show that the terpolymer lubricant additive significantly improves the load-bearing capacity and antifriction ability of the base fluid. According to the test results, it is speculated that the fullerene copolymer nanospheres not only effectively isolate the surfaces of the two friction pairs to improve the bearing capacity and reduce wear, but also generate microelastic rolling lubrication to reduce the friction coefficient. Li et al. [65] used a four-ball machine to investigate the extreme pressure and antiwear properties of C60 additives in liquid paraffin. The results show that C60 additive has good extreme pressure and antiwear performance under higher load and higher speed, and it has high chemical stability.

It can be seen from the above literature that fullerene shows good tribological properties between the friction pairs due to the super molecular structure and good chemical inertness. In addition, fullerene has a certain aromaticity and can be well dissolved in organic solvents. The diameter of the C60 nanospherical particles dissolved in the cutting fluid is very small. Its molecule is a hollow spherical structure. The density of C60 nanoparticles is $1.65 \mathrm{~g} / \mathrm{cm}^{3}$, which is closer to the density of water (density: $1.00 \mathrm{~g} / \mathrm{cm}^{3}$ ) and that of raw cutting oil (density: $0.869 \mathrm{~g} / \mathrm{cm}^{3}$ ). So it is easy to be suspended in the HM32 antiwear lubricating fluid by the dispersion method, and the cutting oil penetrates into the contact surface between the tool and the workpiece or the chip. Meanwhile, the C60 nanoparticles have high compressive strength, and the $\mathrm{C} 60$ nanospherical particles have high thermal stability. The melting point is above $3000^{\circ} \mathrm{C}$. Therefore, it has innate advantages as a lubricant additive. However, the research on its lubrication performance, mechanism, and application is not comprehensive enough.

In this paper, fullerene nanoparticles are used as the additive of HM32 antiwear lubricating fluid to study the dispersion stability and tribological properties. Firstly, the fullerene particles and the Group III base oil are electromagnetically stirred under normal temperature condition to prepare fullerene additives. Then, the fullerene additive and HM32 antiwear lubricating fluid are electromagnetically stirred at room temperature. Finally, the fullerene nanomicrosphere lubricating fluid is ultrasonically oscillated for long time to make the fullerene nanoparticles uniformly dispersed. The dispersion stability of fullerene nanoparticles in HM32 antiwear lubricating fluid was characterized by microscope scanning, precipitation tests, and ultraviolet spectroscopy. Then a four-ball friction tester was used to carry out the friction and wear test. The friction characteristics and extreme pressure performance of 7 kinds of lubricating fluids with fullerene concentration ranging from $100 \mathrm{ppm}$ to $1000 \mathrm{ppm}$ were measured. The experimental results show that fullerene nanoparticle can significantly improve the extreme pressure property and wear resistance of HM32 basic lubricating fluid. The best concentration of fullerene nanoparticles 
is $200 \mathrm{ppm}$. When the fullerene concentration reaches $200 \mathrm{ppm}$, the maximum nonsintering load increases by $36.01 \%$, the sintering load increases by $100.81 \%$, the friction coefficient decreases by $41.28 \%$, and the wear mark diameter of the steel ball decreases by $10.40 \%$. Meanwhile, we found that an excessively high concentration of fullerene nanoparticles will increase the friction and wear of the four-ball friction pair. It may be that too many fullerene nanoparticles are more likely to agglomerate to produce macromolecular fullerenes. It increases the internal friction of lubricating oil molecules and deteriorates the tribological properties of lubricating fluid.

\section{Materials and Methods}

2.1. Fullerene Characterization and Lubricant Additives' Preparation. The fullerene nanoparticles used in this paper are from Xiamen Funa New Material Technology Co., Ltd. The purity of the fullerene solid particles is analyzed and calculated by liquid chromatography. The purity of C60 is calculated to be $99.950 \%$. The equipment used is Shimadzu liquid chromatography SPD-16 by integrating the peak area of HPLC (High-Performance Liquid Chromatography). The chromatogram data of the C60 product and the purity calculation table is shown in Table 1.

The microscopic morphology of fullerene C60 nanoparticles is shown in Figure 1. The photographing equipment used is the ZEISS Gemini SEM 500 field emission scanning electron microscope. It can be seen from the micrograph image that the fullerene solid particles are composed of several spherical fullerene molecular clusters.

We choose Total HM32 construction machinery antiwear hydraulic oil as the base fluid. The HM32 antiwear hydraulic oil has excellent antiwear properties and hydraulic stability. It is widely used in mechanical hydraulic systems and metal cutting systems. Firstly, the fullerene particles and Group III base oil are electromagnetically stirred under normal temperature conditions to prepare fullerene additives. The electromagnetic stirring lasts up to 4 hours to fully disperse the fullerene particles. Then, the fullerene additive and the HM32 antiwear lubricating fluid were continuously electromagnetically stirred at room temperature for 4 hours. Finally, the fullerene nanomicrosphere lubricating fluid was ultrasonically oscillated for 2 hours to make the fullerene nanoparticles uniformly dispersed in the base fluid. We have prepared a total of 6 suspensions with fullerene mass fractions: 100 ppm, 200 ppm, 300 ppm, 400 ppm, 500 ppm, and $1000 \mathrm{ppm}$.

\subsection{Dispersibility and Stability Test of Fullerene} Nanoparticles. We characterized the dispersion stability of fullerene nanoparticles in the HM32 antiwear lubricating fluid through microscope scanning experiment, static sedimentation experiment, and absorption experiment.

2.2.1. Microscope Scanning Experiment. We scan and photograph the nanofullerene particle suspension after electromagnetic stirring and ultrasonic oscillation. The nanofullerene particle suspension is dropped on a clean silicon wafer. The silicon wafers used are ultrasonically shaken and cleaned in an ethanol/acetone mixed solution for one hour, and they are dried in a drying box. We put the silicon wafer dripped with the fullerene suspension on the heating table for a constant temperature heating treatment at 300 degrees Celsius for 2 hours to ensure that the liquid in the suspension evaporates away and the fullerene nanoparticles remain on the silicon wafer. Finally, the dried silicon wafer with fullerene nanoparticles was microscopically photographed. The photographing equipment used is a ZEISS Gemini SEM 500 field emission scanning electron microscope. The actual instrument is shown in Figure 2. By observing the distribution of fullerene nanoparticles, we can analyze the distribution of fullerene nanoparticles in the suspension.

2.2.2. The Static Sedimentation Experiment. The static sedimentation experiment refers to letting the fullerene nanoparticle suspension solution stand still and settle under gravity. The static precipitation method mainly evaluates the stability of the fullerene additive in the lubricating fluid by visually observing the change process of the supernatant liquid height and the precipitation volume. The lower the supernatant height the smaller the volume of the sedimentation. It indicates that the stability of the fullerene additive in the lubricating fluid is better. Conversely, the higher the supernatant height the larger the precipitation volume. It indicates that the stability of the fullerene additive in the lubricating fluid is worse.

The prepared fullerene nanoparticle suspension is placed in a ventilated and dry place. We take pictures of the fullerene nanoparticle suspensions at regular intervals. Afterwards, we observe the precipitation of the suspension with different storage time to analyze the dispersion stability of the fullerene nanoparticles in the suspension.

2.2.3. The Absorption Experiment. The absorption experiment is to measure the absorbance of the fullerene nanoparticle suspension supernatant with an ultraviolet spectrophotometer. The absorbance value is proportional to the number of fullerene nanoparticles in the suspension. Absorbance is defined as

$$
\text { absorbance }=\lg \left(\frac{I_{0}}{I}\right)
$$

where $I_{0}$ stands for the incident light intensity and $I$ stands for the transmitted light intensity.

The higher the absorbance value is, the more the fullerene nanoparticles are contained in the upper solution per unit volume. It means that the dispersion of particles in the suspension is better. Therefore, we use the absorption spectrophotometric method to evaluate the dispersibility of the fullerene additives in the lubricating fluid. The prepared fullerene nanoparticle suspension is placed in an ultravioletvisible spectrometer for spectrum measurement. The instrument used is Shimadzu UV-Vis spectrophotometer2600. The actual instrument is shown in Figure 3. 
TABLE 1: The chromatogram data of the $\mathrm{C} 60$ product and the purity calculation.

\begin{tabular}{lcccccc}
\hline Peak no. & Time & Area & Peak start time & Peak end time & Area (\%) & Remark \\
\hline 1 & 5.110 & 1046 & 4.908 & 5.383 & 0.008 & C60 \\
2 & 8.178 & 13453835 & 7.700 & 10.408 & 9.950 & C60 oxides \\
3 & 9.272 & 892 & 9.117 & 17.325 & 0.007 & 0.035 \\
4 & 16.708 & 4757 & 16.092 & & 100.000 & \\
Total & & 13460529 & & & & \\
\hline
\end{tabular}
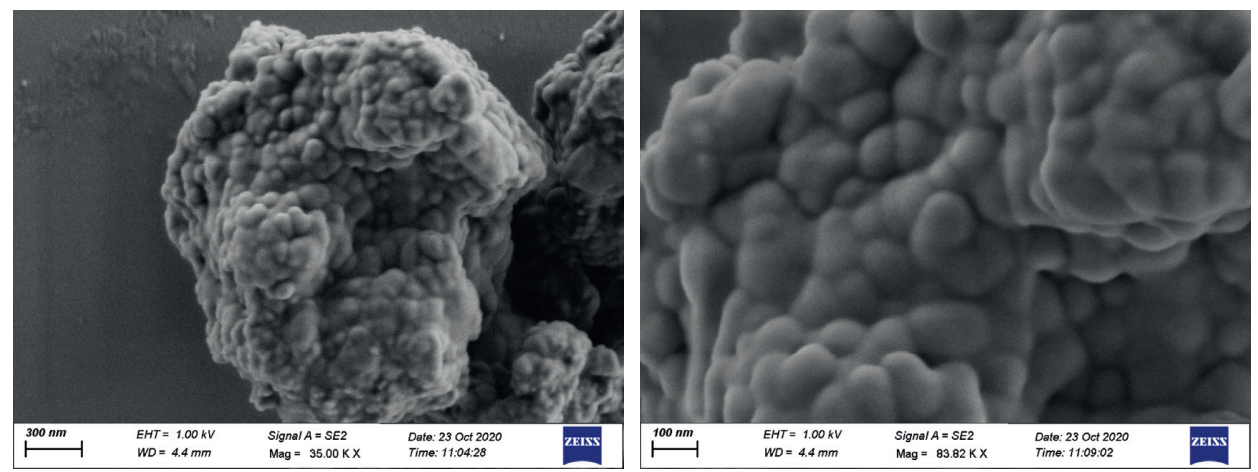

Figure 1: Microscopic morphology of fullerene C60 nanoparticles.

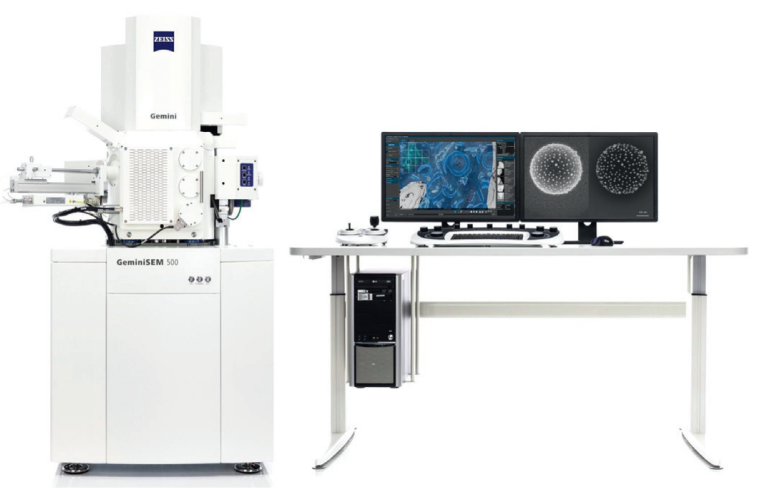

FIgURE 2: ZEISS Gemini SEM 500 field emission scanning electron microscope.

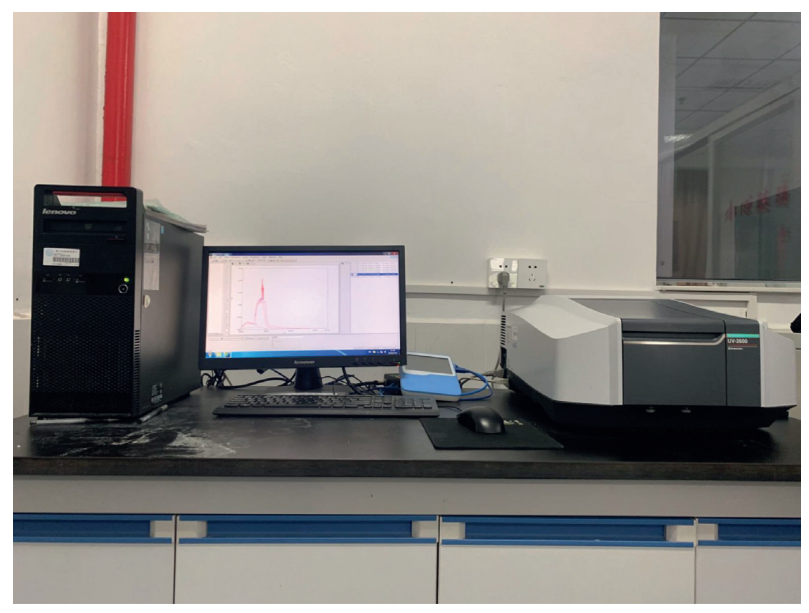

Figure 3: Shimadzu UV-Vis spectrophotometer-2600.
We put the fullerene nanoparticle suspension into a clean spectroscopic dish. The spectrometer was cleaned by ultrasonic shaking in a mixed solution of ethanol/acetone for one hour. Then the spectroscopic dish containing the fullerene nanoparticle suspension was placed into the UV-2600 to obtain the spectrum corresponding to the fullerene nanoparticle suspension. We measure the spectrum of each fullerene nanoparticle suspension in an ultraviolet-visible spectrometer at regular intervals. We observe the change process of the suspension spectrum for different storage time to analyze the dispersion stability of the fullerene nanoparticles in the suspension.

2.3. Friction and Wear Test of Fullerene Nanoparticles. We use the Xiamen Tianji MS-10J four-ball friction testing machine to carry out the friction and wear test of the C60 nanoparticle suspension. The four-ball friction machine is a point contact tester, which can accurately measure the wear value in a short time and requires only a small sample amount. The four-ball machine can measure the friction properties of lubricating oils, as well as extreme pressure and wear properties, including lubrication properties that protect the friction pair from scratches, seizures, sintering, or other wear phenomena under heavy loads. Therefore, the four-ball friction tester is widely used in the determination of the antiwear performance and load-bearing capacity of lubricants.

The physical map of the equipment is shown in Figure 4. The testing machine adopts the load control mode of automatic loading and realizes stepless speed regulation through the servo motor. The friction pair used in this article is a four-ball friction pair, and the main structure diagram is shown in Figure 5. 


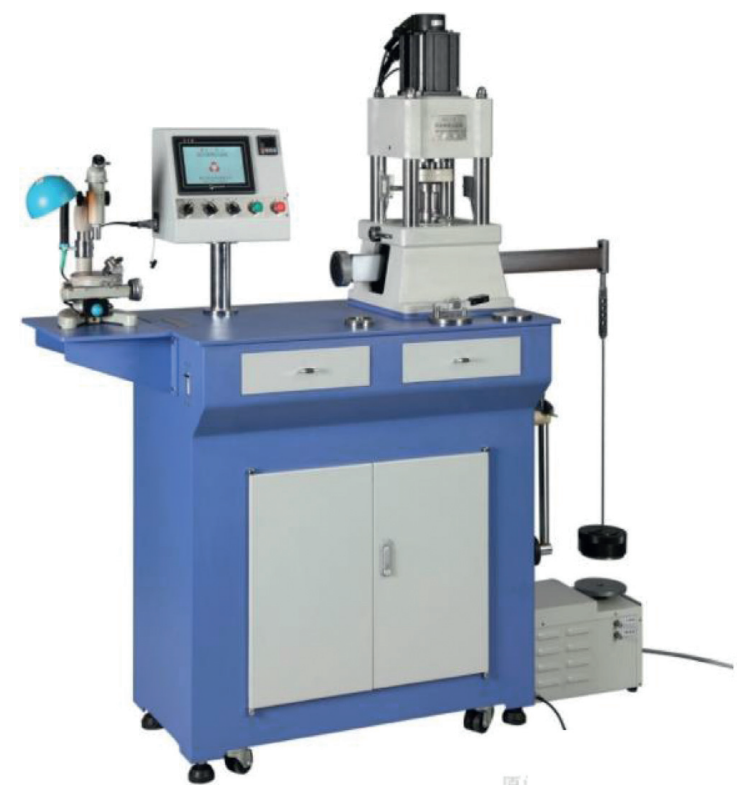

FIGURE 4: Four-ball friction testing machine MS-10J (produced by Xiamen Tianji Automation Co., Ltd.).

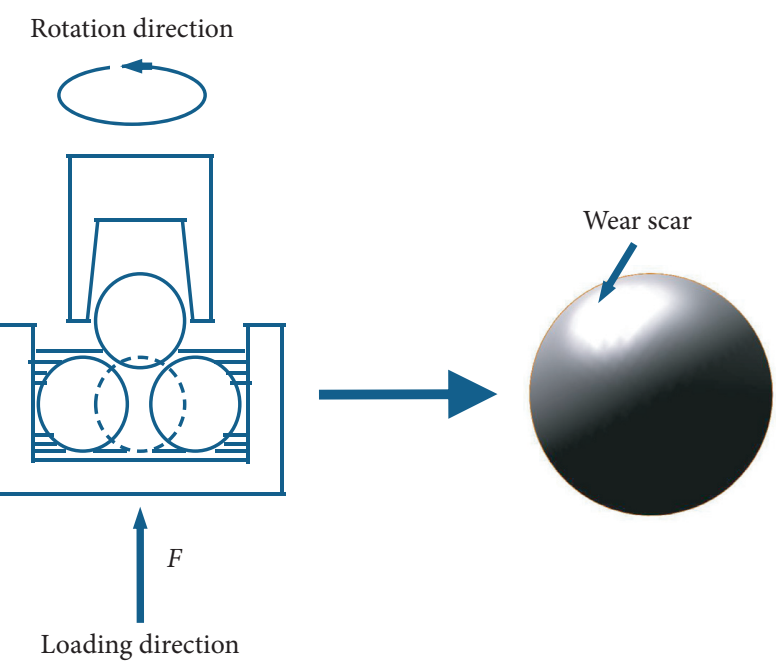

Figure 5: Schematic diagram of four-ball friction and wear tester device.

The three steel balls in the lower oil box are in contact with each other, and the steel balls in the upper clamp are in point contact with the three steel balls. The friction pair is made of GCr15, the diameter is $12.70 \mathrm{~mm}$, and the hardness is HRC60. Before starting the experiment, the fullerene nanoparticle suspension was poured into the oil box below. Therefore, the steel balls can be completely immersed in the test solution. We operated the numerical control system to control the loading load of the lower fixture and the loading speed of the upper fixture when all four steel balls are in contact. In this way, friction can be generated between the friction pairs and wear spots can be obtained on the three steel balls. The steel ball wear and sintering tests were carried out by the four-ball friction tester to evaluate the largest nonseizure load $\mathrm{PB}$, sintering load $\mathrm{PD}$, and wear scar diameter of the steel ball friction pair under the fullerene suspension lubrication.

\section{Results and Discussion}

3.1. Study on Dispersion Stability of Fullerene Nanomicrosphere Lubricating Additives. The microscopic morphology of $300 \mathrm{ppm}$ fullerene nanoparticle suspension is shown in Figure 6. From the microscopic morphology of the fullerene nanoparticle suspension, it can be seen that the fullerene nanoparticles are relatively uniformly distributed on the silicon wafer. The fullerene nanoparticles present agglomerated spherical shape. The oil molecules show ring shape structure. What is more, the molecular film formed by the fullerene nanomolecules and oil molecules is uniformly mixed together. It preliminarily shows that the fullerene nanoparticles are uniformly dispersed in the HM32 base fluid.

We place the prepared fullerene nanoparticle suspension in a ventilated and dry environment for static sedimentation. The lubricating fluids with seven fullerene concentrations after standing for 10 days and 20 days are shown in Figure 7. It can be seen from Figure 7 that there is still no clear liquid in the upper region of the fullerene nanoparticle suspension and no precipitation in the lower region after standing for 20 days. Each bottle of fullerene nanoparticle suspension maintains a uniform color. It preliminarily shows that the prepared fullerene nanoparticle suspension can maintain a uniform distribution of fullerene nanoparticles over time and has excellent dispersion stability.

Figure 8 shows the UV spectrum of 7 kinds of lubricating fluids with fullerene concentration ranging from $100 \mathrm{ppm}$ to $1000 \mathrm{ppm}$. It can be seen from Figure 8 that the UV spectrum of the fullerene nanoparticle suspension after standing for 20 days is very similar to the original UV spectrum. It shows that the concentration of fullerene nanoparticles in the upper region of the fullerene nanoparticle suspension does not change significantly. It indicates that the prepared fullerene nanoparticle suspension can maintain a uniform distribution of fullerene nanoparticles for a long time and has excellent dispersion stability.

\subsection{Study on Friction Characteristics of C60 Nanomicrosphere Lubricating Additives}

3.2.1. Extreme Pressure Performance of Fullerene Nanoparticles in HM32 Base Fluid. In order to comprehensively investigate the extreme pressure performance of fullerene nanoparticles in the HM32 base fluid, we measured the maximum seizure-free load and sintering load of seven kinds of lubricating fluids with different fullerene concentrations ranging from $100 \mathrm{ppm}$ to $1000 \mathrm{ppm}$. The results are shown in Table 2. It can be seen from Table 2 that fullerene nanoparticles have a significant impact on the extreme pressure performance of the HM32 base fluid. When the fullerene concentration reached $200 \mathrm{ppm}$, the maximum nonseize load increased by $36.01 \%$ and the sintering load increased by $100.81 \%$. But when the concentration is increased to more than $200 \mathrm{ppm}$, the maximum nonjamming load is basically maintained at the 


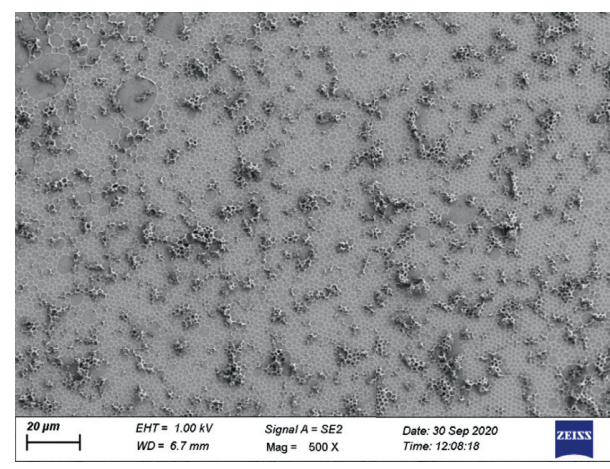

(a)

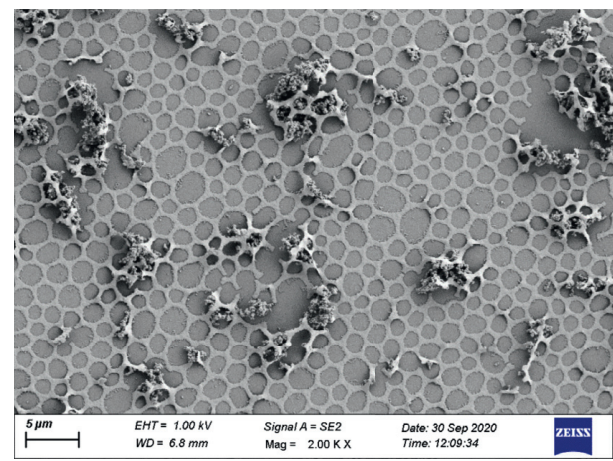

(c)

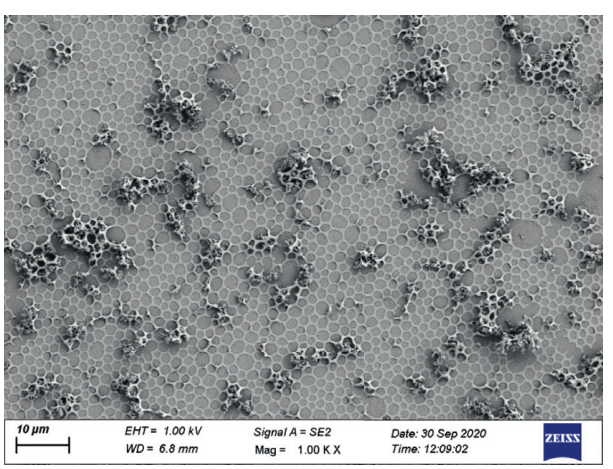

(b)

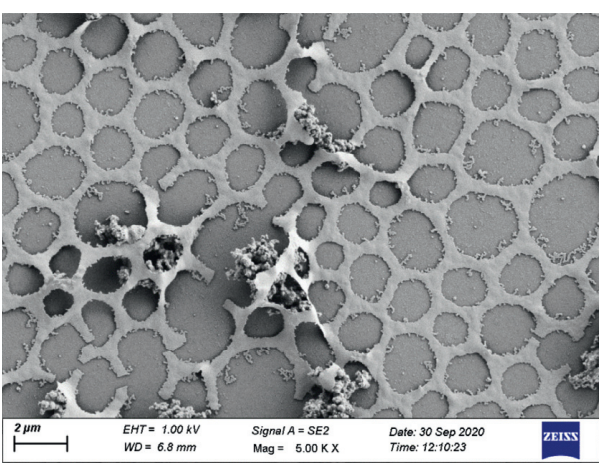

(d)

FIgURE 6: Microscopic morphology of $300 \mathrm{ppm}$ fullerene nanoparticle suspension.

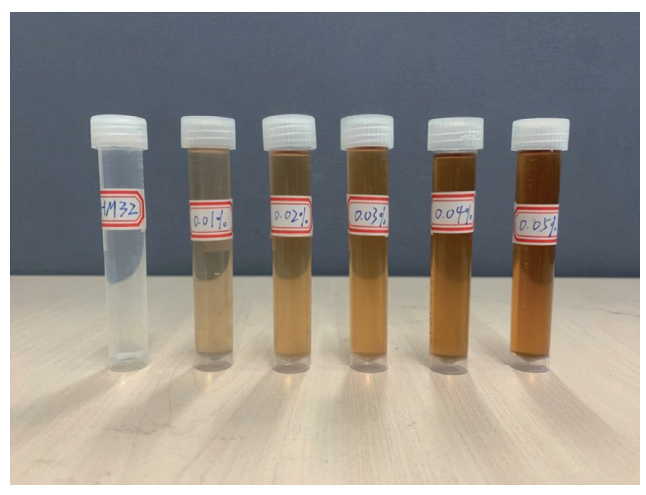

(a)

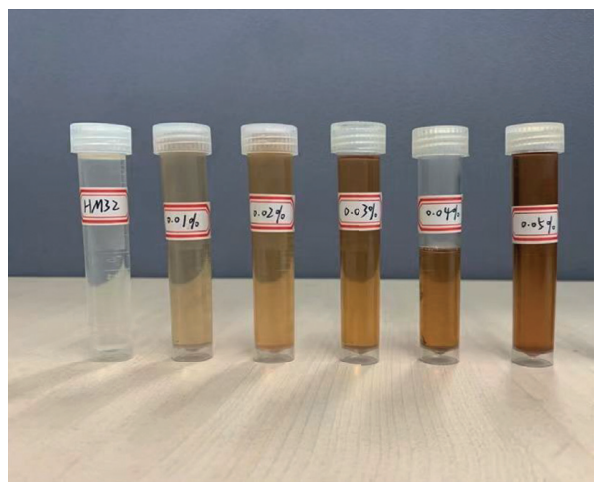

(b)

FIgURE 7: Fullerene nanoparticle suspension: (a) left for 10 days and (b) left for 20 days.

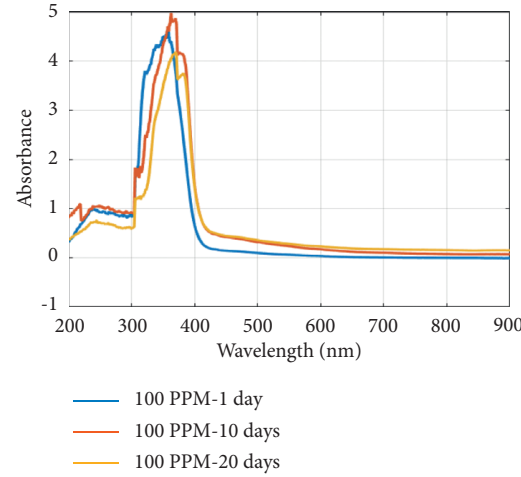

(a)

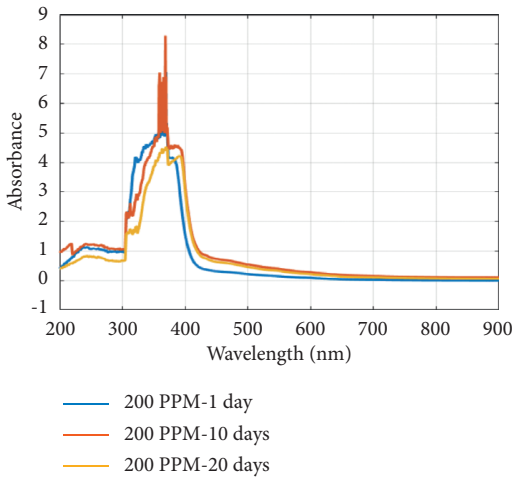

(b)

Figure 8: Continued. 


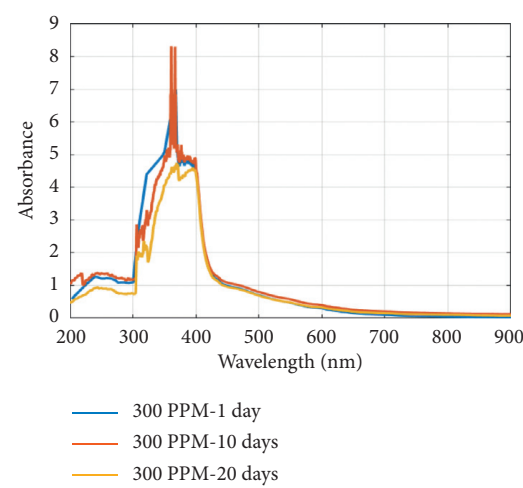

(c)

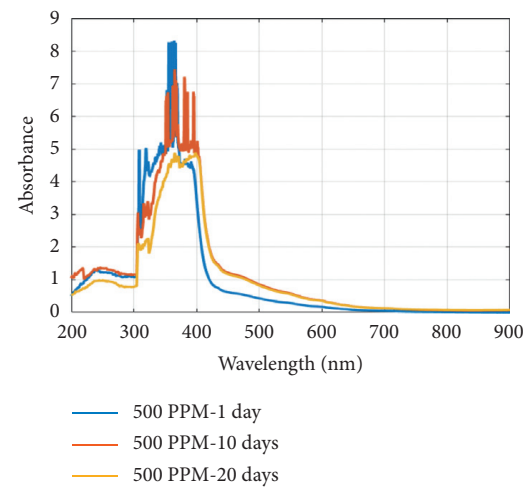

(e)

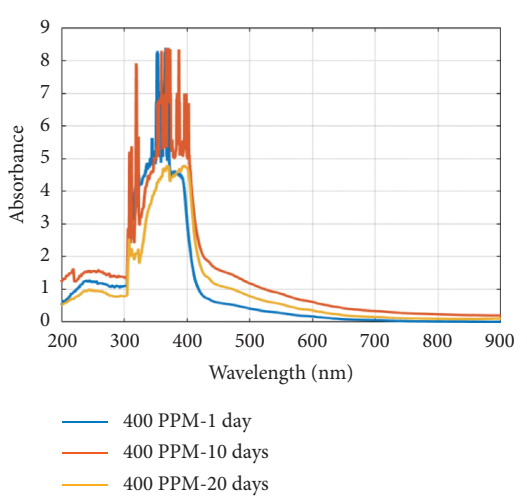

(d)

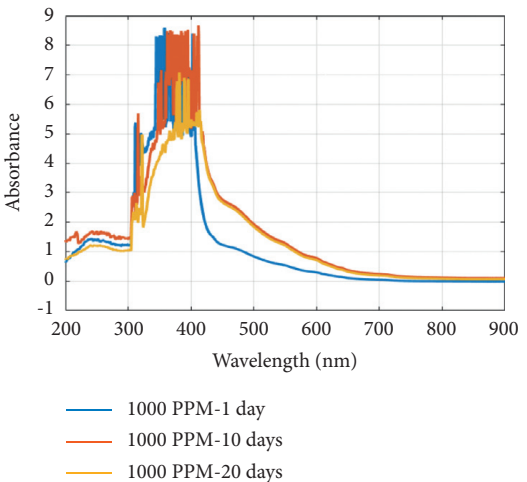

(f)

FIGURE 8: The UV spectrum of fullerene nanoparticles lubricating fluids.

TABLE 2: Extreme pressure properties of fullerene nanoparticles in the HM32 base oil.

\begin{tabular}{lcc}
\hline Lubricating fluid & The largest nonseizure load (PB) (N) & The sintering load (PD) (N) \\
\hline HM32 & 647 & 1235 \\
HM32 + 100 ppm C60 nanoparticle & 765 & 1782 \\
HM32 + 200 ppm C60 nanoparticle & 880 & 2480 \\
HM32 + 300 ppm C60 nanoparticle & 892 & 2504 \\
HM32 + 400 ppm C60 nanoparticle & 901 & 2513 \\
HM32 + 500 ppm C60 nanoparticle & 900 & 2528 \\
HM32 + 1000 ppm C60 nanoparticle & 912 & 2550 \\
\hline
\end{tabular}

same level, and the increase rate of the sintering load is significantly reduced. As the concentration increases, the extreme pressure performance has not been further improved. It indicates fullerene nanoparticles can significantly improve the extreme pressure performance of lubricants. However, it is difficult to further improve the extreme pressure performance of lubricating base fluid when the fullerene nanoparticles concentration exceeds a certain amount.

3.2.2. Friction Properties of Fullerene Nanoparticles in HM32 Base Fluid. In order to comprehensively investigate the extreme pressure performance of fullerenes in the HM32 base fluid, the friction coefficient and steel ball wear scar diameter (WSD) of seven fullerene lubricating fluids after a four-ball long grinding test under a $200 \mathrm{~N}$ normal load were measured. The results are shown in Table 3 . It can be seen from Table 3 that fullerenes show significant antiwear and antiwear properties. Especially when the concentration is $200 \mathrm{ppm}$, the friction coefficient and the diameter of the wear scar of the steel ball are significantly improved. When the fullerene concentration reached $200 \mathrm{ppm}$, the friction coefficient decreased by $41.28 \%$ and the wear scar diameter of the steel ball decreased by $10.40 \%$. However, when the concentration increased to more than $200 \mathrm{ppm}$, the friction coefficient and steel ball wear scar diameter did not continue to significantly decrease or even fluctuate, and the antiwear and antiwear performance were not further improved. It indicates that a proper concentration of fullerene nanoparticles can significantly improve the antiwear and antiwear properties of the HM32 base fluid, but an excessively high concentration of fullerene nanoparticles will increase friction and wear. It may be that too many fullerene nanoparticles are more likely to agglomerate to produce 
TABLE 3: Frication properties of fullerene nanoparticles in the HM32 base oil (normal load $200 \mathrm{~N}$ ).

\begin{tabular}{lcc}
\hline Lubricating fluid & Frication coefficient & The wear scar diameter $(\mathrm{mm})$ \\
\hline HM32 & 0.109 & 0.519 \\
HM32 + 100 ppm C60 nanoparticle & 0.086 & 0.498 \\
HM32 + 200 ppm C60 nanoparticle & 0.064 & 0.465 \\
HM32 + 300 ppm C60 nanoparticle & 0.069 & 0.447 \\
HM32 + 400 ppm C60 nanoparticle & 0.078 & 0.487 \\
HM32 + 500 ppm C60 nanoparticle & 0.067 & 0.509 \\
HM32 + 1000 ppm C60 nanoparticle & 0.071 & 0.513 \\
\hline
\end{tabular}

macromolecular fullerenes, which cannot form an adsorption film on the friction surface. It will also increase the internal friction of the lubricating oil molecules, making the tribological properties of the lubricating oil worse.

\section{Conclusions}

In order to explore the application prospects of fullerene lubricating additives, this paper thoroughly studies the dispersion stability and tribological property of fullerene nanoparticles in lubricating fluids. In this paper, a fullerene nanoparticle suspension was prepared by a combination of electrolytic stirring and ultrasonic dispersion. The dispersion stability of fullerene nanoparticles in HM32 antiwear lubricating fluid was comprehensively studied by microscope scanning experiment, static sedimentation experiment, and absorption experiment. Finally, the reliability of the preparation method for fullerene nanoparticle suspension was verified. The four-ball friction experiment method was used to investigate the extreme pressure and tribological properties of 7 kinds of lubricating fluids with fullerene concentration ranging from $100 \mathrm{ppm}$ to $1000 \mathrm{ppm}$. The results show that fullerene nanoparticle can significantly improve the extreme pressure performance and wear resistance of HM32 basic lubricating fluid.

Through the research of this paper, the following conclusions can be drawn:

(1) The combination of electromagnetic stirring and ultrasonic dispersion is a very effective process to maintain the dispersion stability of fullerene nanoparticles in the lubricating base liquid. It can keep the fullerene nanoparticle suspension in a stable state for a long time without precipitation.

(2) Fullerene nanoparticles have a significant improvement effect on the extreme pressure performance of HM32 base fluid. The maximum nonsintering load increases by $36.01 \%$ and the sintering load increases by $100.81 \%$ when the fullerene concentration reaches $200 \mathrm{ppm}$. However, it is difficult to further improve the extreme pressure performance of the HM32 lubricating base fluid when the fullerene nanoparticles concentration exceeds $200 \mathrm{ppm}$.

(3) Fullerene has significant antiwear property. The friction coefficient decreases by $41.28 \%$ and the steel ball wear scar diameter decreases by $10.40 \%$ when the fullerene concentration reaches $200 \mathrm{ppm}$. However, an excessively high concentration of fullerene nanoparticles will increase friction and wear. It may be that too many fullerene nanoparticles are more likely to agglomerate to produce macromolecular fullerenes. It increases the internal friction of lubricating oil molecules and deteriorates the tribological properties of lubricating oil.

\section{Data Availability}

The data used to support the findings of this study are available from the corresponding author upon request.

\section{Conflicts of Interest}

The authors declare that the research was conducted in the absence of any commercial or financial relationships that could be construed as a potential conflict of interest.

\section{Authors' Contributions}

Jingshan Huang and Bin Yao conceived the lubricating additives; Jingshan Huang and Hao Sun performed the experiment; Jingshan Huang preprocessed and analyzed the data; Jingshan Huang wrote the paper; Xi Wang and Binqiang Chen reviewed and edited the manuscript; all authors read and approved the manuscript.

\section{References}

[1] J. Luo and J. Li, "The progress and future of tribology," $\mathrm{Lu}$ brication \& Sealing, vol. 12, pp. 1-12, 2010.

[2] I. El-Thalji and E. Jantunen, "A summary of fault modelling and predictive health monitoring of rolling element bearings," Mechanical Systems and Signal Processing, vol. 60-61, pp. 252-272, 2015.

[3] L. Jing, M. Zhao, P. Li, and X. Xu, "A convolutional neural network based feature learning and fault diagnosis method for the condition monitoring of gearbox," Measurement, vol. 111, pp. 1-10, 2017.

[4] J. Ou, H. Li, G. Huang, and G. Yang, "Intelligent analysis of tool wear state using stacked denoising autoencoder with online sequential-extreme learning machine," Measurement, vol. 167, Article ID 108153, 2020.

[5] Q. Nazir and C. Shao, "Online tool condition monitoring for ultrasonic metal welding via sensor fusion and machine learning," Journal of Manufacturing Processes, vol. 62, no. 5, pp. 806-816, 2021.

[6] P. D. Deshpande, B. P. Gautham, A. Cecen, S. Kalidindi, A. Agrawal, and A. Choudhary, "Application of statistical and machine learning techniques for correlating properties to composition and manufacturing processes of steels," in 
Proceedings of the 2nd World Congress on Integrated Computational Materials Engineering (ICME), 2013.

[7] B.-q. Chen, B.-x. Zheng, C.-q. Wang, and W.-f. Sun, "Adaptive sparse detector for suppressing powerline component in EEG measurements," Frontiers in Public Health, vol. 9, Article ID 669190, 2021.

[8] B. Chen, B. Zheng, and W. Sun, "Ultra-resolution spectral correction based on adaptive linear neuron for biomedical signal processing," Frontiers in Public Health, vol. 9, Article ID 682377, 2021.

[9] B. Chen, Y. Li, W. Sun, and W. He, "Removal of power line interference from ECG signals using adaptive notch filters of sharp resolution," IEEE Access, vol. 7, pp. 150667-150676, 2019.

[10] A. M. Khorasani and A. Kootsookos, "Modeling and optimization of the cutting fluid flow and parameters for increasing tool life in slot milling on st52," International Journal of Modeling Simulation \& Scientific Computing, vol. 4, no. 2, pp. 270-326, 2013.

[11] F. Wei, K. Zhang, S. Cai, C. Sun, W. Sun, and B. Liu, “A force model for face grinding using digital graphic scanning (DGS) method," International Journal of Advanced Manufacturing Technology, vol. 113, pp. 3261-3270, 2021.

[12] F. J. Alonso and D. R. Salgado, "Analysis of the structure of vibration signals for tool wear detection," Mechanical Systems and Signal Processing, vol. 22, no. 3, pp. 735-748, 2008.

[13] W. Song, S. Deng, J. Yang, and Q. Cheng, "Tool wear detection based on duffing-holmes oscillator," Mathematical Problems in Engineering, vol. 2008, Article ID 510406, 15 pages, 2008.

[14] S. Dutta, A. Datta, N. D. Chakladar, S. K. Pal, S. Mukhopadhyay, and R. Sen, "Detection of tool condition from the turned surface images using an accurate grey level co-occurrence technique," Precision Engineering, vol. 36, no. 3, pp. 458-466, 2012.

[15] A. Siddhpura and R. Paurobally, "A review of flank wear prediction methods for tool condition monitoring in a turning process," International Journal of Advanced Manufacturing Technology, vol. 65, no. 1-4, pp. 371-393, 2013.

[16] W. Sun, H. Gao, S. Tan, Z. Wang, and L. Duan, "Wear detection of WC-Cu based impregnated diamond bit matrix based on SEM image and deep learning," International Journal of Refractory Metals and Hard Materials, vol. 98, Article ID 105530, 2021.

[17] P. Junior, D’Addona, M. Doriana, and P. R. Aguiar, “Dressing tool condition monitoring through impedance-based sensors: Part 1-PZT diaphragm transducer response and EMI sensing technique," Sensors, vol. 18, no. 12, 2018.

[18] M. D. Prieto, G. Cirrincione, A. G. Espinosa, J. A. Ortega, and H. Henao, "Bearing fault detection by a novel conditionmonitoring scheme based on statistical-time features and neural networks," IEEE Transactions on Industrial Electronics, vol. 60, no. 8, pp. 3398-3407, 2013.

[19] A. Fatemeh, T. Antoine, and T. Marc, "Tool condition monitoring using spectral subtraction and convolutional neural networks in milling process," International Journal of Advanced Manufacturing Technology, vol. 98, pp. 3217-3227, 2018.

[20] D. Azad and K. Ramji, "Identification of bearing assembly defects using finite element analysis and condition monitoring techniques," International Journal of Engineering Research \& Technology, vol. 1, pp. 1-5, 2012.

[21] H. O. A. Ahmed, M. L. D. Wong, and A. K. Nandi, "Intelligent condition monitoring method for bearing faults from highly compressed measurements using sparse over-complete features," Mechanical Systems and Signal Processing, vol. 99, pp. 459-477, 2018.

[22] Y. Huang and Y. Shin, "Method of intelligent fault diagnosis of preload loss for single nut ball screws through the sensed vibration signals," International Journal of Mechanical and Mechatronics Engineering, vol. 6, no. 5, 2012.

[23] P. C. Tsai, C. C. Cheng, and Y. C. Hwang, "Ball screw preload loss detection using ball pass frequency," Mechanical Systems and Signal Processing, vol. 48, no. 1-2, pp. 77-91, 2014.

[24] T. R. Lin, E. Kim, and A. C. C. Tan, "A practical signal processing approach for condition monitoring of low speed machinery using peak-hold-down-sample algorithm," $M e$ chanical Systems and Signal Processing, vol. 36, no. 2, pp. 256-270, 2013.

[25] J. Huang, B. Chen, L. Yang, and W. Sun, "Fractal geometry of wavelet decomposition in mechanical signature analysis," Measurement, vol. 173, no. 5, Article ID 108571, 2020.

[26] A. K. Sharma, R. K. Singh, A. R. Dixit, and A. K. Tiwari, "Novel uses of alumina-MoS2 hybrid nanoparticle enriched cutting fluid in hard turning of AISI 304 steel," Journal of Manufacturing Processes, vol. 30, pp. 467-482, 2017.

[27] S. E. Mustafa, M. Ali, A. Iqbal, M. B. N. Shaikh, and R. Hassan, "Formulation and analysis of cost-effective environmentfriendly metal cutting nanofluids using zinc oxide on turning of AISI 52100 steel using MQL," Engineering Research Express, vol. 3, 2020.

[28] X. Sun, The Influence of Graphene and Carbon Nanotubes on the Friction Properties of Lubricants, Harbin Institute of Technology, Harbin, China, 2017.

[29] V. Vasu and K. Kumar, "Analysis of nanofluids as cutting fluid in grinding EN-31 steel," Nano-Micro Letters, vol. 3, no. 4, pp. 209-214, 2011.

[30] S. Wen, Nanotribology, Tsinghua University Press, Beijing, China, 1998.

[31] G. Duursma, K. Sefiane, and A. Kennedy, "Experimental studies of nanofluid droplets in spray cooling," Heat Transfer Engineering, vol. 30, no. 13, pp. 1108-1120, 2009.

[32] E. G. Zadoshenko, V. E. Burlakova, and A. A. Novikova, "Effect of nickel nanopowder on lubrication behaviour of lowtemperature grease in steel-steel tribosystem," Tribology: Materials, Surfaces \& Interfaces, vol. 14, no. 1, pp. 1-8, 2019.

[33] G.-Z. Zhu, Y.-Z. Gao, S.-Y. Liu, and H.-C. Zhang, "Antiwear and self-repairing mechanisms of magnesium hydroxysilicate composite powder as a lubricating oil additive for steel-steel pair," Journal of Tribology, vol. 32, no. 2, pp. 183-188, 2012.

[34] Q. Wan, Yi Jin, P. Sun, and Y. Ding, "Rheological and tribological behaviour of lubricating oils containing platelet $\mathrm{MoS}_{2}$ nanoparticles," Journal of Nanoparticle Research, vol. 16, no. 5, pp. 1-9, 2014.

[35] S. Kwon, J.-H. Ko, K.-J. Jeon, Y.-H. Kim, and J. Y. Park, "Enhanced nanoscale friction on fluorinated graphene," Nano Letters, vol. 12, no. 12, pp. 6043-6048, 2012.

[36] X. Yan, Study on Dispersion Stability and Tribological Properties of Nano-Serpentine/GO in Base Lubricating Oil, Yanshan University, Qinhuangdao, China, 2019.

[37] H. Liu, S.-M. Zhang, P.-Y. Zhang, and Y.-J. Zhang, "Preparation of bismuth nanoparticles via a thermal decomposition process and evaluation of their tribological properties in synthetic ester oils," in Proceedings of the 14th IFToMM World Congress, pp. 547-555, Taipei, Taiwan, 2015.

[38] S. H. Musavi, B. Davoodi, and S. A. Niknam, "Effects of reinforced nanofluid with nanoparticles on cutting tool wear morphology," Journal of Central South University, vol. 26, no. 5, pp. 1050-1064, 2019. 
[39] H. Xie, Study on the Tribological Properties and Lubricant Mechanisms of Nanolubricants for Magnesium Alloys Rolling, Chongqing University, Chongqing, China, 2016.

[40] F. Jian and Y. Zhao, "Wear self-compensating tribological effects of lubricating additives," Materials Protection, vol. 39, pp. 34-36, 2006.

[41] C. Zhang, S. Zhang, S. Song et al., "Preparation and tribological properties of surface-capped copper nanoparticle as a water-based lubricant additivee," Tribology Letters, vol. 54, no. 1, pp. 25-33, 2014.

[42] S. Ingole, A. Charanpahari, K. Amol, S. S. Umareb, D. V. Bhat, and J. Menghani, "Tribological behavior of nano $\mathrm{TiO}_{2}$ as an additive in base oil," Wear, vol. 301, no. 1-2, pp. 776-785, 2013.

[43] T. Luo, X. Wei, L. Huang, and F. Yang, "Tribological properties of $\mathrm{Al}_{2} \mathrm{O}_{3}$ nanoparticles as lubricating oil additives," Ceramics International, vol. 40, no. 5, pp. 7143-7149, 2014.

[44] M. Gulzar, H. Masjuki, M. Varman et al., "Improving the AW/ EP ability of chemically modified palm oil by adding $\mathrm{CuO}$ and $\mathrm{MoS}_{2}$ nanoparticles," Tribology International, vol. 88, pp. 271-279, 2015.

[45] R. Chou, A. H. Battez, J. J. Cabello, J. L. Viesca, A. Osorio, and A. Sagastume, "Tribological behavior of polyalphaolefin with the addition of nickel nanoparticles," Tribology International, vol. 43, no. 12, pp. 2327-2332, 2010.

[46] S. Yang, X.-J. Wang, X.-F. Li, and X. Li, "Influence of pH and SDBS on the stability and thermal conductivity of nanofluids," Energy \& Fuels, vol. 23, no. 5, pp. 270-278, 2009.

[47] Z. Jia, P. Wang, Y. Xia, H.-b. Zhang, X. Pang, and B. Li, "Tribological behaviors of diamond-like carbon coatings on plasma nitrided steel using three BN-containing lubricants," Applied Surface Science, vol. 255, no. 13-14, pp. 6666-6674, 2009.

[48] L. Imene, D. Fabrice, V. Beatrice, and M. Jean-Michel, "Real time TEM imaging of compression and shear of single fullerene-like $\mathrm{MoS}_{2}$ nanoparticle," Tribology Letters, vol. 45, pp. 131-141, 2011.

[49] D. X. Peng, Y. Kang, R. M. Hwang, S. S. Shyr, and Y. P. Chang, "Tribological properties of diamond and $\mathrm{SiO}_{2}$ nanoparticles added in paraffin," Tribology International, vol. 42, no. 6, pp. 911-917, 2009.

[50] Y. Xia, W. Yang, X. Ma et al., "Research on the improvement of anti-wear properties of lubricating oil by nanometer copper powder," Lubrication and Sealing, vol. 5, pp. 43-44, 1998.

[51] Y.-H. Xu, G.-B. Yang, S.-M. Zhang, and P.-Y. Zhang, "Preparation of water-soluble nano-copper and its tribological properties," Acta Tribology, vol. 32, no. 2, pp. 165-170, 2012.

[52] L. Zhao, Z. Cai, Z. Zhang, and X. Zhang, "Tribological properties of graphene as effective lubricant additive in oil on textured bronze surface," Chinese Journal of Materials Research, vol. 30, no. 1, pp. 57-62, 2016.

[53] M. K. Gupta, P. K. Sood, and V. S. Sharma, "Optimization of machining parameters and cutting fluids during nano-fluid based minimum quantity lubrication turning of titanium alloy by using evolutionary techniques," Journal of Cleaner Production, vol. 135, pp. 1276-1288, 2016.

[54] R. Padmini, P. V. Krishna, and G. K. M. Rao, "Effectiveness of vegetable oil based nanofluids as potential cutting fluids in turning AISI 1040 steel," Tribology International, vol. 94, pp. 490-501, 2016.

[55] A. K. Sharma, A. K. Tiwari, A. R. Dixit, R. K. Singh, and M. Singh, "Novel uses of alumina/graphene hybrid nanoparticle additives for improved tribological properties of lubricant in turning operation," Tribology International, vol. 119, pp. 99-111, 2018.

[56] M. H. Cetin and S. K. Kilincarslan, "Effects of cutting fluids with nano-silver and borax additives on milling performance of aluminium alloys," Journal of Manufacturing Processes, vol. 50, pp. 170-182, 2020.

[57] L. Hong, Study on the Synthesis and Tribological Properties of Water-Soluble Fullerene Copolymer Nano-Microsphere Lubricating additives, Huazhong University of Science and Technology, Wuhan, China, 2001.

[58] L. Hong, J. Luo, and X. Hu, "Tribological research progress of fullerene (C60)," Lubrication and Sealing, vol. 27, no. 1, pp. 31-33, 2002.

[59] D. G. Tochil'nikov, "Effect of fullerene black additives on boundary sliding friction of steel counterbodies lubricated with mineral oil," Journal of Friction and Wear, vol. 33, no. 2, pp. 94-100, 2012.

[60] J. Lee, "Enhancement of lubrication properties of nano-oil by controlling the amount of fullerene nanoparticle additives," Tribology Letters, vol. 28, pp. 203-208, 2007.

[61] A. Hirata, M. Igarashi, and T. Kaito, "Study on solid lubricant properties of carbon onions produced by heat treatment of diamond clusters or particles," Tribology International, vol. 37, no. 11-12, pp. 899-905, 2004.

[62] Y. Yao, X. Wang, and J. Guo, "Tribological property of onionlike fullerenes as lubricant additive," Materials Letters, vol. 62, pp. 2524-2527, 2008.

[63] F. Yan, Z. Jin, and X. Zhang, "Study on the tribological properties of C60/C70 as lubricant additives," Tribology Journal, vol. 13, no. 1, pp. 59-62, 1993.

[64] L. Hong, W. Guan, and D. Liao, "Tribological behavior of fullerene-styrene-maleic anhydride terpolymer," Applied Chemistry, vol. 17, no. 2, pp. 180-182, 2000.

[65] J. Li, Li Han, and W. Sun, "Research on the tribological properties of C60," Chinese Journal of Tribology, vol. 20, no. 4, pp. 307-309, 2000. 